\title{
Transient Phenomena in Quantum Bound States Subjected to a Sudden Perturbation
}

\author{
Marcos MOSHINSKY and Emerson SADURNÍ \\ Instituto de Física, Universidad Nacional Autónoma de México, Apartado Postal 20-364, \\ 01000 México D.F., México \\ E-mail:moshi@fisica.unam.mx,sadurni@fisica.unam.mx
}

Received June 24, 2005, in final form August 08, 2005; Published online August 18, 2005

Original article is available at http://www.emis.de/journals/SIGMA/2005/Paper003/

\begin{abstract}
Transient phenomena in quantum mechanics have been of interest to one of the authors (MM) since long ago and, in this paper, we focus on the problem of a potential $V_{-}$ which for negative times gives rise to bound states and is suddenly changed at $t=0$ to a potential $V_{+}$which includes $V_{-}$plus a perturbed term. An example will be the deuteron (where the proton and neutron are assumed to interact through an oscillator potential) submitted to a sudden electrostatic field. The analysis for $t>0$ can be carried out with the help of appropriate Feynmann propagators and we arrive at the result that the separation between the nucleons has an amplitude that depends on the intensity of the electrostatic field, but its period continues to be related with the inverse of the frequency of the oscillator proposed for the interaction. A general approximate procedure for arbitrary problems of this type is also presented at the end.
\end{abstract}

Key words: transient phenomena; propagators

2000 Mathematics Subject Classification: 81V35; 81Q05

\section{Introduction}

The word "Transient" will mean for us here that the expectation values of measurable observable or transition probabilities in quantum mechanics will be time dependent, in contrast with the "stationary" case in which they are not. From the very origin of quantum mechanics, in the ninety twenties, these transient phenomena could be discussed through the time dependent versions of the equations of motion in the Heisenberg or Schrödinger picture.

The most natural way of discussing the transient phenomena is through the version of quantum mechanics that Feynman [1] proposed in the ninety forties. For him the fundamental concept was a propagator, also sometimes called Feynmans kernel, that relates an initial state characterized by the wave function $\psi\left(\boldsymbol{x}^{\prime}, t^{\prime}\right)$ (where $\boldsymbol{x}^{\prime}$ is a notation for all the coordinates of the system and $t^{\prime}$ its time) with the final state $\psi(\boldsymbol{x}, t)$ with $t>t^{\prime}$. This propagator was denoted by $K\left(\boldsymbol{x}, t ; \boldsymbol{x}^{\prime}, t^{\prime}\right)$ and Feynman gives a procedure to calculate it starting from a Lagrangian formulation (in terms of generalized coordinates and velocities) of the classical problem and summing all paths suggested by the action principle between $\boldsymbol{x}^{\prime}$ and $\boldsymbol{x}$. If the classical Lagrangian, is not explicitly dependent on time, the propagator only depends on the difference of the times i.e. $t-t^{\prime}$ so, without loss of generality, we could take $t^{\prime}=0$ and write

$$
K\left(\boldsymbol{x}, t ; \boldsymbol{x}^{\prime}, 0\right) \equiv K\left(\boldsymbol{x}, t ; \boldsymbol{x}^{\prime}\right), \quad \psi\left(\boldsymbol{x}^{\prime}, 0\right) \equiv \psi\left(\boldsymbol{x}^{\prime}\right)
$$


so that we have

$$
\psi(\boldsymbol{x}, t)=\int K\left(\boldsymbol{x}, t, \boldsymbol{x}^{\prime}\right) \psi\left(\boldsymbol{x}^{\prime}\right) d \boldsymbol{x}^{\prime}
$$

which implies that the propagator $K\left(\boldsymbol{x}, t, \boldsymbol{x}^{\prime}\right)$ satisfies a time dependent Schrödinger equation with the initial condition

$$
K\left(\boldsymbol{x}, 0 ; \boldsymbol{x}^{\prime}\right)=\delta\left(\boldsymbol{x}-\boldsymbol{x}^{\prime}\right) .
$$

The use of the propagator concept has been enhanced by the appearance a few years ago of a "Handbook of Feynman Path Integrals" [2], and the power of the concept, in the case of a one dimensional free particle, is illustrated in the Appendix for a compact derivation of the problem of "Diffraction in time", which one of the authors (MM) [3] analyzed long ago by other methods.

The general type of problems that we wish to discuss here is that of the potential $V_{-}(\boldsymbol{x})$ which for negative times $t<0$ is one that admits bound states and that at $t=0$ is suddenly changed to a potential $V_{+}(\boldsymbol{x})$ that contains $V_{-}(\boldsymbol{x})$ plus some type of external interaction given by $V_{+}(\boldsymbol{x})-V_{-}(\boldsymbol{x})$. If our vector $\boldsymbol{x}$ is given in cartesian coordinates the kinetic energy is a sum of terms $\left(m_{s} \dot{x}_{q s}^{2}\right)$ where $q=1, \ldots, m$ is the index for the dimension $m$ of our single particle vector space and $s=1, \ldots, n$ the index for the number of particles. Thus our classical Lagrangian is well defined and we can look through the tables of reference [2] to see whether the propagator is available. We thus proceed to analyze the following problem.

\section{The deuteron subjected to a sudden electrostatic field}

The first physical problem that came to our attention along the lines of the last paragraph of the previous section, was the hydrogen atom in a sudden constant electrostatic field. Unfortunately the propagator for the Coulomb problem alone is already quite complicated, and more so if a linear electrostatic potential is added.

We thus wanted to focus on problems in which the propagator can be fully and simply determined, and that led us to Lagrangians that are quadratic in the coordinate and velocities variables, of which there is an extensive list of propagators in reference [2].

Why are quadratic Lagrangian (which imply also quadratic Hamiltonians in the coordinate and momentum variables) give propagators of the Gaussian type [2]? One answer is given in a paper by one of the authors (MM) and C. Quesne [4]. The time evolution associated with a classical Lagrangian is given by a canonical transformation which conserves a symplectic metric. For quadratic Lagrangians or Hamiltonian this canonical transformation is linear and it provides the dynamical group of the problem. To translate it to quantum mechanics we have to take its unitary representation and this gives a Gaussian propagator [4]. An example of this problem is the Lagrangian or Hamiltonian of the harmonic oscillator.

Thus we were led to the deuteron which we can consider, as is usually done in nuclear physics, as a system of neutron and proton, of essentially the same mass $m$ and interacting through an harmonic oscillator potential of frequency $\omega$.

With units in which $\hbar=m=c=1$ ( $c$ being the velocity of light so that everything will be dimensionless) and if we apply suddenly at $t=0$ an electrostatic field, in the direction $z$, acting solely on the proton, the Lagrangian will be

$$
\mathcal{L}=\frac{1}{2}\left(\dot{\boldsymbol{r}}_{1}+\dot{\boldsymbol{r}}_{2}^{2}\right)-\frac{1}{2} \omega^{2}\left(\boldsymbol{r}_{1}-\boldsymbol{r}_{2}\right)^{2}-\mathcal{E} z_{1}
$$

where the indexes 1 and 2 will correspond respectively to the proton and neutron and $\mathcal{E}$ is in our units the intensity of a linear potential between the plates of a condenser multiplied by the charge of the proton. 
We proceed now to consider an orthogonal transformation leading essentially to relative and center of mass coordinates

$$
\boldsymbol{r}=\frac{1}{\sqrt{2}}\left(\boldsymbol{r}_{1}-\boldsymbol{r}_{2}\right), \quad \boldsymbol{R}=\frac{1}{\sqrt{2}}\left(\boldsymbol{r}_{1}+\boldsymbol{r}_{2}\right)
$$

which transforms $\mathcal{L}$ to

$$
\mathcal{L}=\left(\frac{1}{2} \dot{\boldsymbol{r}}^{2}-\frac{1}{2} \omega^{2} \boldsymbol{r}^{2}-\frac{\mathcal{E}}{\sqrt{2}} z\right)+\left(\frac{1}{2} \dot{\boldsymbol{R}}^{2}-\frac{\mathcal{E}}{\sqrt{2}} Z\right)
$$

where the components of the vectors $\boldsymbol{r}$ and $\boldsymbol{R}$ are indicated respectively as

$$
\boldsymbol{r}=\boldsymbol{i} x+\boldsymbol{j} y+\boldsymbol{k} z, \quad \boldsymbol{R}=\boldsymbol{i} X+\boldsymbol{j} Y+\boldsymbol{k} Z
$$

with $\boldsymbol{i}, \boldsymbol{j}, \boldsymbol{k}$ being unit vectors in the directions indicated.

If a Lagrangian can be expressed as a sum of two terms depending on different observables the propagator is given by the product of the propagators associated with these two terms. Furthermore, as the squares of the vectors $\boldsymbol{r}, \dot{\boldsymbol{R}}$ are given by

$$
r^{2}=x^{2}+y^{2}+z^{2}, \quad \dot{R}^{2}=\dot{X}^{2}+\dot{Y}^{2}+\dot{Z}^{2}
$$

it is convenient to express our problem in cartesian coordinates where the Lagrangian (2) becomes

$$
\begin{aligned}
\mathcal{L}= & {\left[\frac{1}{2}\left(\dot{x}^{2}-\omega^{2} x^{2}\right)+\frac{1}{2}\left(\dot{y}^{2}-\omega^{2} y^{2}\right)+\frac{1}{2}\left(\dot{z}^{2}-\omega^{2} z^{2}-\sqrt{2} \mathcal{E} z\right)\right] } \\
& +\left[\frac{1}{2} \dot{X}^{2}+\frac{1}{2} \dot{Y}^{2}+\frac{1}{2}\left(\dot{Z}^{2}-\sqrt{2} \mathcal{E} Z\right)\right] .
\end{aligned}
$$

Thus, from the observation at the beginning of the previous paragraph, the propagator associated with $\mathcal{L}$ will be the product of the one dimensional propagators associated with the six terms appearing in (3). For $\frac{1}{2}\left(\dot{x}^{2}-\omega^{2} x^{2}\right)$ the propagator is given in [2, p. 178, formula (6.2.33)] as

$$
\left[\frac{\omega}{2 \pi i \sin \omega t}\right]^{\frac{1}{2}} \exp \left\{-\frac{\omega}{2 i}\left[\left(x^{2}+x^{\prime 2}\right) \cot \omega t-\frac{2 x x^{\prime}}{\sin \omega t}\right]\right\}
$$

For $\frac{1}{2}\left(\dot{y}^{2}-\omega^{2} y^{2}\right)$ the propagator is again of the form (4) but $x, x^{\prime}$ replaced by $y, y^{\prime}$. For $\frac{1}{2}\left(\dot{z}^{2}-\omega^{2} z^{2}-\sqrt{2} \mathcal{E} z\right)$ we complete the square introducing the variable $\bar{z}$ by

$$
\bar{z}=z+\left(\mathcal{E} / \sqrt{2} \omega^{2}\right)
$$

and thus the one dimensional Lagrangian becomes

$$
\frac{1}{2}\left(\dot{\bar{z}}^{2}-\omega^{2} \bar{z}^{2}\right)+\frac{\mathcal{E}^{2}}{4 \omega^{2}}
$$

as $\dot{\bar{z}}=\dot{z}$. Furthermore for the constant term $\left(\mathcal{E}^{2} / 4 \omega^{2}\right)$ we can apply the relation between propagators and Green functions of the time dependent Schrödinger equation, mentioned in p. 2 of reference [2], to see that it contributes the phase term $\exp \left[-i\left(\mathcal{E}^{2} / 4 \omega^{2}\right) t\right]$ while the remainder in (6) is just the one-dimensional Lagrangian of the oscillator for the propagator of which we can use equation (4) replacing $x, x^{\prime}$ by $\bar{z}, \bar{z}^{\prime}$. It is convenient to express the propagator involving the relative coordinates in terms of only barred variables if we add the definitions

$$
\bar{x}=x, \quad \bar{y}=y
$$


as in these coordinates we have only the oscillator potential for $x$ and $y$. With the definitions

$$
\bar{r}^{2}=\bar{x}^{2}+\bar{y}^{2}+\bar{z}^{2}, \quad \overline{\boldsymbol{r}}=\boldsymbol{i} \bar{x}+\boldsymbol{j} \bar{y}+\boldsymbol{k} \bar{z}
$$

and the previous discussion concerning the phase factor associated with $\left(\mathcal{E}^{2} / 4 \omega^{2}\right)$ we obtain then that the part of the propagator related to the relative coordinate $\overline{\boldsymbol{r}}$ becomes

$$
\exp \left[-i\left(\mathcal{E}^{2} / 4 \omega^{2}\right) t\right]\left[\frac{\omega}{2 \pi i \sin \omega t}\right]^{3 / 2} \exp \left\{-\frac{\omega}{2 i}\left[\left(\bar{r}^{2}+\bar{r}^{2}\right) \cot \omega t-\frac{2 \overline{\boldsymbol{r}} \cdot \overline{\boldsymbol{r}}^{\prime}}{\sin \omega t}\right]\right\} \text {. }
$$

Turning now to the expression (3) with capital letters we start with $\frac{1}{2} \dot{X}^{2}$ which from reference $[2$, p. 174, formula $(6.2 .10)]$ becomes

$$
\frac{1}{(2 \pi i t)^{\frac{1}{2}}} \exp \left[\frac{i}{2 t}\left(X-X^{\prime}\right)^{2}\right] \text {. }
$$

For $\frac{1}{2} \dot{Y}^{2}$ it is the same formula (8) but with $X, X^{\prime}$ replaced by $Y, Y^{\prime}$. For $\left(\frac{1}{2} \dot{Z}^{2}-\frac{\mathcal{E}}{\sqrt{2}} Z\right)$ we use reference $[2$, p. 175 , formula $(6.2 .18)]$ to get

$$
\frac{1}{(2 \pi i t)^{\frac{1}{2}}} \exp \left\{i\left[\frac{\left(Z-Z^{\prime}\right)^{2}}{2 t}-\frac{\mathcal{E} t}{2 \sqrt{2}}\left(Z+Z^{\prime}\right)-\frac{\mathcal{E}^{2} t^{3}}{48}\right]\right\} \text {. }
$$

Multiplying (8), the corresponding expression for $Y, Y^{\prime}$, and (9) we get for the center of mass part of the Lagrangian the expression

$$
\frac{1}{(2 \pi i t)^{3 / 2}} \exp \left\{i\left[\frac{\left(\boldsymbol{R}-\boldsymbol{R}^{\prime}\right)^{2}}{2 t}-\frac{\mathcal{E} t}{2 \sqrt{2}}\left(Z+Z^{\prime}\right)-\frac{\mathcal{E}^{2} t^{3}}{48}\right]\right\} \text {. }
$$

Now the full propagator associated with the Lagrangian (3) will be the product of (7) and (10).

For a deuteron at $t>0$ the ground state is

$$
A \exp \left(-\frac{1}{2} \omega r^{2}\right) \exp (i \boldsymbol{K} \cdot \boldsymbol{R})
$$

where $A$ is the normalization of the Gaussian term given by

$$
A=(\omega / \pi)^{3 / 4} .
$$

If we want to know these wave function at time $t$ we have to apply the propagator associated to (3) to the initial state (11) which we proceed to do in the next section.

\section{The wave function for the deuteron at $t>0$ in a suddenly applied electrostatic field}

As we have obtained now the propagator (7), (10) associated with the Lagrangian (3), we can apply it to the state (11) to get through equation (1) the state at a given time $t>0$.

The calculation involves integrals of exponentials of quadratic expressions in the variables $\overline{\boldsymbol{r}}^{\prime}$ and $\boldsymbol{R}^{\prime}$ which can be carried out by completing squares in the exponents. We will calculate explicitly one example and then give the final result for $\psi(\boldsymbol{r}, \boldsymbol{R}, t)$ when $t>0$.

Remembering that for the $x$ component of the relative vector $\boldsymbol{r}$ we have the propagator (4), when we apply it to the part of the initial wave function $\exp \left(-\frac{1}{2} \omega x^{2}\right)$ of (11) we have to evaluate the integral

$$
\begin{gathered}
\int_{-\infty}^{\infty} A^{1 / 3}\left(\frac{\omega}{2 \pi i \sin \omega t}\right)^{1 / 2} \exp \left\{\frac{i \omega}{2}\left[\left(x^{2}+x^{\prime 2}\right) \cot \omega t-2 x x^{\prime}(\sin \omega t)^{-1}\right]\right\} e^{-\frac{1}{2} \omega x^{\prime 2}} d x^{\prime} \\
=A^{1 / 3}\left(\frac{\omega}{2 \pi i \sin \omega t}\right)^{\frac{1}{2}} \exp \left(\frac{i \omega}{2} x^{2} \cot \omega t\right) \int_{-\infty}^{\infty} e^{-\beta x^{\prime 2}+\alpha x^{\prime}} d x^{\prime},
\end{gathered}
$$


where

$$
\beta=\frac{\omega}{2}(1-i \cot \omega t), \quad \alpha=-i x \omega(\sin \omega t)^{-1}
$$

and the real part of $\beta$ is positive. From reference [5] we have that

$$
\int_{-\infty}^{\infty} \exp \left(-\beta x^{2}+\alpha x^{\prime}\right) d x^{\prime}=(\pi / \beta)^{\frac{1}{2}} \exp \left(\alpha^{2} / 4 \beta\right)
$$

and from (13) we obtain

$$
\beta=\frac{\omega}{2 i}(\sin \omega t)^{-1} \exp (i \omega t), \quad \frac{\alpha^{2}}{4 \beta}=i \frac{\omega}{2} x^{2} \cot \omega t-\frac{1}{2} \omega x^{2}
$$

so that replacing in (14) and then in (12) we obtain

$$
A^{1 / 3} \exp \left(-\frac{1}{2} \omega x^{2}\right) \exp \left(-\frac{i \omega t}{2}\right)
$$

which, as we should expect is, in our units, the phase term associated with the energy $(\omega / 2)$ of the ground state of the oscillator.

The same result holds for the $y$ variable and both $x, y$ can be replaced by $\bar{x}, \bar{y}$, as the electrostatic potential is applied only in the $z$ direction.

For the case when our initial wave function is $A^{1 / 3} \exp \left(-\omega z^{2} / 2\right)$, it is best to replace in it $z$ by $\left[\bar{z}-\left(\mathcal{E} / \sqrt{2} \omega^{2}\right)\right]$, as indicated in (5), and use for the variable $\bar{z}$ the propagator of the oscillator to which only a time dependent phase factor is multiplied as discussed after equation (6).

With transition to the center of mass coordinates of the deuteron, denoted by capital letters, the propagator is just that of the free particle when we have $X, Y$ so applying it to the corresponding part of the plane wave $\exp \left(i K_{X}\right), \exp \left(i K_{y} Y\right)$ they will only give the phase factor

$$
\exp \left(i K_{x} X\right) \exp \left(-i K_{x}^{2} t / 2\right), \quad \exp \left(i K_{y} Y\right) \exp \left(-i K_{y}^{2} t / 2\right) .
$$

For the $Z$ center of mass coordinate we have to apply to $\exp \left(i K_{z} Z\right)$ the propagator (9) and the evaluation of the integral again follows procedures similar to those indicated in equations (12) to $(15)$.

Combining then all our results we can say that for $t>0$ our wave function $\psi(\boldsymbol{r}, \boldsymbol{R}, t)$ will become

$$
\begin{aligned}
\psi(\boldsymbol{r}, \boldsymbol{R}, t)= & A\left(e^{-\frac{1}{2} m \omega r^{2}} e^{-i 3 / 2 \omega t}\right) \exp \left[i\left(\boldsymbol{K} \cdot \boldsymbol{R}-\frac{1}{2} K^{2} t\right)\right] \exp \left(-\frac{i \mathcal{E}^{2} t}{4 \omega^{2}}\right) \\
& \times \exp \left\{\frac{\mathcal{E}}{\sqrt{2} \omega}\left(e^{-i \omega t}-1\right) z+\frac{\mathcal{E}^{2}}{2 \omega^{3}}\left[e^{-i \omega t}-\frac{1}{2}\left(1+\cos ^{2} \omega t\right)\right]+\frac{i \sin 2 \omega t}{4}\right\} \\
& \times \exp \left\{-i\left[\frac{\mathcal{E} t^{3}}{12}+K_{z} \frac{\mathcal{E} t^{2}}{2 \sqrt{2}}-\frac{Z \mathcal{E} t}{\sqrt{2}}\right]\right\} .
\end{aligned}
$$

\section{The probability density for the deuteron in a suddenly applied electrostatic field}

If we turn to the "Diffraction in time" problem discussed in the Appendix, we note that to get information on its time dependent behavior we need not the wave function but its absolute value squared i.e. the probability density. Once we have this we can discuss the behavior in time with the help of the Cornu spiral [3]. This of course is a general procedure in quantum mechanics and thus starting from the wave function in (16) we need to write down $|\psi(\boldsymbol{r}, \boldsymbol{R}, t)|^{2}$. 
We see immediately that all part associated with the center of mass coordinates, i.e. capital $X, Y, Z$, disappears, as it would also disappear for the wave function at time $t=0$ given by (11).

For the relative coordinates we have to keep in the exponent of (16) only the terms that are real multiplied by 2 , so we get

$$
\begin{aligned}
|\psi(\boldsymbol{r}, \boldsymbol{R}, t)|^{2} & =A^{2} \exp \left\{-\omega r^{2}-\frac{2 \mathcal{E}}{\sqrt{2} \omega}(1-\cos \omega t)-\frac{\mathcal{E}^{2}}{2 \omega^{3}}\left[-2 \cos \omega t+\left(1+\cos ^{2} \omega t\right)\right]\right\} \\
& =A^{2} \exp \left[-\omega\left(x^{2}+y^{2}\right)\right] \exp \left\{-\omega\left[z^{2}+\frac{2 \mathcal{E}}{\sqrt{2} \omega}(1-\cos \omega t) z+\frac{\mathcal{E}^{2}}{2 \omega^{4}}(1-\cos \omega t)^{2}\right]\right\} \\
& =A^{2} \exp \left[-\omega\left(x^{2}+y^{2}\right)\right] \exp \left\{-\omega\left[z+\frac{\mathcal{E}}{\sqrt{2} \omega}(1-\cos \omega t)\right]^{2}\right\} \\
& =A^{2} \exp \left[-\omega\left(x^{2}+y^{2}\right)\right] \exp \left\{-\omega\left[z+\frac{\sqrt{2} \mathcal{E}}{\omega} \sin ^{2}(\omega t / 2)\right]^{2}\right\},
\end{aligned}
$$

where used a trigonometric relation for the last expression.

We have thus a very simple transient behavior that depends only on the relative coordinates and in which $x, y$ have the standard Gaussian behavior of range $\omega^{-1 / 2}$ in our units, while $z$ oscillates around this range with an amplitude $\left(\sqrt{2} \mathcal{E} / \omega^{2}\right) \sin ^{2}(\omega t / 2)$. The period of oscillation is

$$
(\omega T / 2)=\pi \quad \text { or } \quad T=(2 \pi / \omega)
$$

while the amplitude is $\left(\sqrt{2} \mathcal{E} / \omega^{2}\right)$.

Note that coordinates of proton and neutron, in the direction of the electrostatic field, are given respectively by

$$
z_{1}=\frac{1}{\sqrt{2}}(Z+z), \quad z_{2}=\frac{1}{\sqrt{2}}(Z-z)
$$

so if we take our origin at the position of the center of mass, the distance between proton and neutron goes as $\sqrt{2} z$ and thus the deuteron is vibrating with an amplitude proportional to $\left(2 \mathcal{E} / \omega^{2}\right) \sin ^{2}(\omega t / 2)$ and it could radiate for strong electrostatic fields.

Clearly for very strong electrostatic fields the deuteron could desintegrate and this information can be obtained also from the discussion of the classical version of the problem.

The information available for propagators in reference [2] would allow us to discuss also the problem of a time dependent electrostatic field which reflect more the physical situation. The formulas we could use (6.2.42) is in p. 180 of reference [2].

For a constant electromagnetic field given by a vector potential $\boldsymbol{A}$ we could use formula (6.2.17) of p. 175 of reference [2].

In general Feynman procedure gives a powerful tool for analyzing transient phenomena when external fields are applied to bound systems.

\section{General procedure for dealing with transient phenomena caused by a sudden perturbation}

While the propagator $K\left(\boldsymbol{x}, t ; \boldsymbol{x}^{\prime}\right)$ is very useful for the discussion of transient phenomena, it is not usually available for the general potential $V_{+}(\boldsymbol{x})$. It is thus useful to develop an approximate procedure that is always available. 
Denoting for $H_{ \pm}$the Hamiltonians associated with the potential $V_{ \pm}$we have that

$$
H_{-}=T+V_{-}, \quad H_{+}=T+V_{+}=H_{-}+\left(V_{+}-V_{-}\right)
$$

where $T$ is the kinetic energy.

We shall assume that $H_{-}$has only a discrete spectrum. In the case it also has a continuous spectrum we can reduce it to the previous case by inserting the whole system into a box. We shall denote the energies and eigenfunctions of $H_{-}$as

$$
H_{-} \phi_{n}=E_{n} \phi_{n}
$$

where

$$
E_{0} \leq E_{1} \leq E_{2} \leq \cdots \leq E_{n-1} \leq E_{n} \leq E_{n+1} \cdots
$$

For $H_{+}$we are in search of a solution $\psi(\boldsymbol{x}, t)$ of the time dependent equation (in c.g.s. units)

$$
i \hbar \frac{\partial \psi}{\partial t}=H_{+} \psi=\left[H_{-}+\left(V_{+}-V_{-}\right)\right] \psi
$$

with the initial value

$$
\psi(\boldsymbol{x}, 0)=\phi_{\nu}(\boldsymbol{x})
$$

where $\nu$ is some integer.

We define the Laplace transform of $\psi(\boldsymbol{x}, t)$ by

$$
\bar{\psi}(\boldsymbol{x}, s)=\int_{0}^{\infty} e^{-s t} \psi(\boldsymbol{x}, t) d t .
$$

Applying the transform to equation (17) we get

$$
\begin{aligned}
i \hbar \int_{0}^{\infty} e^{-s t} \frac{\partial \psi}{\partial t} d t & =i \hbar \int_{0}^{\infty} \frac{\partial}{\partial t}\left(e^{-s t} \psi(\boldsymbol{x}, t)\right) d t+i \hbar s \bar{\psi}(\boldsymbol{x}, s) \\
& =-i \hbar \psi(\boldsymbol{x}, 0)+i \hbar s \bar{\psi}(\boldsymbol{x}, s)=\left[H_{-}+\left(V_{+}-V_{-}\right)\right] \bar{\psi}(\boldsymbol{x}, s) .
\end{aligned}
$$

Now the eigenstates $\phi_{n}$ of $H_{-}$are a complete orthonormal set so we can make the expansion

$$
\bar{\psi}(\boldsymbol{x}, s)=\sum_{n=0}^{\infty} a_{n}(s) \phi_{n}(\boldsymbol{x})
$$

so that equation (18) becomes

$$
-i \hbar \phi_{\nu}(\boldsymbol{x})+i \hbar s \sum_{n} a_{n}(s) \phi_{n}(\boldsymbol{x})=\left[H_{-}+\left(V_{+}-V_{-}\right)\right] \sum_{n} a_{n}(s) \phi_{n}(\boldsymbol{x}) .
$$

Multiplying by $\psi_{n^{\prime}}^{*}(\boldsymbol{x})$ and integrating over the variables $\boldsymbol{x}$ we get

$$
-i \hbar \delta_{n^{\prime} \nu}+i \hbar s a_{n^{\prime}}(s)=E_{n^{\prime}} a_{n^{\prime}}(s)+\sum_{n}\left\langle n^{\prime}\left|V_{+}-V_{-}\right| n\right\rangle a_{n}(s)
$$

where

$$
\left\langle n^{\prime}\left|V_{+}-V_{-}\right| n\right\rangle=\int \phi_{n^{\prime}}^{*}(\boldsymbol{x})\left[V_{+}(\boldsymbol{x})-V_{-}(\boldsymbol{x})\right] \phi_{n}(\boldsymbol{x}) d \boldsymbol{x} .
$$


The equation (19) corresponds to an infinite set of linear algebraic equations for the coefficients $a_{n}(s)$. We can solve them with the usual assumption that after a given index $N$ all coefficients vanish, and then we get the $a_{n}(s)$ for $n=0,1, \ldots, N$ from which we can write the $\bar{\psi}(\boldsymbol{x}, s)$ as

$$
\bar{\psi}(\boldsymbol{x}, s)=\sum_{n=0}^{N} a_{n}(s) \phi_{n}(\boldsymbol{x}) .
$$

We want though to determine $\psi(\boldsymbol{x}, t)$ for which we need the reciprocal of the Laplace transform

$$
\psi(\boldsymbol{x}, t)=\frac{1}{2 \pi i} \int_{c-i \infty}^{c+i \infty} \bar{\psi}(\boldsymbol{x}, s) e^{s t} d s=\frac{1}{2 \pi \hbar} \int_{-\infty+i \hbar c}^{\infty+i \hbar c} \exp (-i E t / \hbar) \bar{\psi}(\boldsymbol{x},-i E / \hbar) d E,
$$

where we replace the variable $s$ by

$$
s=-i(E / \hbar)
$$

with $E$ being integrated along a line above all poles of the function $\bar{\psi}\left(\boldsymbol{x},-i E \hbar^{-1}\right)$. Note that the contour can be completed with a circle from below and get essentially the residues at values of $E$ given by the homogeneous set of linear equations

$$
\left(E-E_{n^{\prime}}\right) a_{n^{\prime}}=\sum_{n}\left\langle n^{\prime}\left|V_{+}-V_{-}\right| n\right\rangle a_{n}
$$

or

$$
E a_{n^{\prime}}=\sum_{n}\left\langle n^{\prime}\left|H_{+}\right| n\right\rangle a_{n}
$$

which essentially give us the energies associated with the levels of the Hamiltonian $H_{+}$.

\section{A Diffraction in time}

Units

$$
\begin{aligned}
& \hbar=m=c=1, \\
& u(l)=(\hbar / m c), \quad u(t)=\left(\hbar / m c^{2}\right), \quad u(m)=m .
\end{aligned}
$$

Free particle propagator

$$
K\left(x, t ; x^{\prime}, 0\right)=(2 \pi i t)^{-\frac{1}{2}} \exp \left[(i / 2 t)\left(x-x^{\prime}\right)^{2}\right] .
$$

The integral for diffraction in time is

$$
\begin{aligned}
M(x, k, t) & =\int_{-\infty}^{0} K\left(x, t ; x^{\prime}, 0\right) \exp \left(i k x^{\prime}\right) d x^{\prime} \\
& =(2 \pi i t)^{-\frac{1}{2}} \exp \left\{i\left(k x-\frac{1}{2} k^{2} t\right)\right\} \int_{-\infty}^{0} \exp \left\{\frac{i}{2 t}\left[x^{\prime}+(k t-x)\right]^{2}\right\} d x^{\prime},
\end{aligned}
$$

where the last expression comes from completing the square for $x^{\prime}$.

We introduce the variable $u$ by the definition

$$
(\pi / 2)^{\frac{1}{2}} u=(2 t)^{-\frac{1}{2}}\left[x^{\prime}+(k t-x)\right]
$$


from which it follows that for $x^{\prime}=0$, we have $u=u_{0}$

$$
u_{0}=(\pi t)^{-\frac{1}{2}}(k t-x), \quad \sqrt{\pi / 2} d u=(2 t)^{-\frac{1}{2}} d x
$$

and thus $M(x, k, t)$ becomes

$$
M(x, k, t)=e^{-i \pi / 4} \exp \left[i\left(k x-\frac{1}{2} k^{2} t\right)\right]\left[\int_{-\infty}^{0} \exp \left(i \pi u^{2} / 2\right) d u+\int_{0}^{u_{0}} \exp \left(i \pi u^{2} / 2\right) d u\right] .
$$

But we have [5]

$$
\int_{-\infty}^{0} \exp \left(i \pi u^{2} / 2\right) d u=\frac{(1+i)}{2}, \quad \int_{0}^{u_{0}} \exp \left(i \pi u^{2} / 2\right) d u=C\left(u_{0}\right)+i S\left(u_{0}\right)
$$

with the definition of Fresnel integrals

$$
\begin{aligned}
& C\left(u_{0}\right)=\int_{0}^{u_{0}} \cos \left(\pi u^{2} / 2\right) d u, \quad S\left(u_{0}\right)=\int_{0}^{u_{0}} \sin \left(\pi u^{2} / 2\right) d u \\
& M(x, k, t)=e^{-i \pi / 4} \exp \left[i\left(k x-\frac{1}{2} k^{2} t\right)\right]\left\{\left[\frac{1}{2}+C\left(u_{0}\right)\right]+i\left[\frac{1}{2}+S\left(u_{0}\right)\right]\right\}
\end{aligned}
$$

and

$$
|M(x, k, t)|^{2}=\left\{\left[\frac{1}{2}+C\left(u_{0}\right)\right]^{2}+\left[\frac{1}{2}+S\left(u_{0}\right)\right]^{2}\right\}
$$

for which the Cornu spiral can be used to show the difference between the quantum behavior given by (20) and the classical one in which the probability density is 1 in the interval $\infty \leq x \leq v t$ and 0 for $v t \leq x \leq \infty[3]$.

[1] Feynman R.P., Space-time approach to non-relativistic quantum mechanics, Rev. Mod. Phys., 1948, V.20, 367-387.

[2] Grosche C., Steiner F., Handbook of Feynman path integrals, Springer Tracts in Modern Physics, V.145, Berlin, Springer-Verlag, 1998.

[3] Moshinsky M., Diffraction in time, Phys. Rev., 1952, V.88, 625-631.

[4] Moshinsky M., Quesne C., Linear transformations and their unitary representation, J. Math. Phys., 1971, V.12, 1772-1780.

[5] Gradshteyn I.S., Ryzhik I.M., Tables of integrals, series and products, New York - London, Academic Press, 1965. 